\title{
Optical spectroscopy of DPVs and the case of LP Ara
}

\section{Ronald E. Mennickent ${ }^{1}$, Darek Graczyk ${ }^{1}$, Zbigniew Kołaczkowski ${ }^{2}$, Gabriela Michalska ${ }^{2}$, Daniela Barría ${ }^{1}$ and Ewa Niemczura ${ }^{2}$}

${ }^{1}$ Dpto. de Astronomía, Universidad de Concepción, Chile, ${ }^{2}$ Instytut Astronomiczny Uniwersytetu Wrocławskiego, Wrocław, Poland

\begin{abstract}
We present preliminary results of our spectroscopic campaign of a group of intermediate mass interacting binaries dubbed "Double Periodic Variables" (DPVs), characterized by orbital light curves and additional long photometric cycles recurring roughly after 33 orbital periods (Mennickent et al. 2003, 2005). They have been interpreted as interacting, semi-detached binaries showing cycles of mass loss into the interstellar medium (Mennickent et al. 2008, Mennickent \& Kołaczkowski 2009). High resolution Balmer and helium line profiles of DPVs can be interpreted in terms of mass flows in these systems. A system solution is given for LP Ara, based on modeling of the ASAS V-band orbital light curve and the radial velocity of the donor star.
\end{abstract}

Keywords. stars: binaries, stars: early-type

\section{Spectra of Galactic DPVs and report on the analysis of LP Ara}

During recent years we have monitored a sample of Galactic DPVs with high resolution optical spectrographs. From the inspection of the spectral region around $\mathrm{H} \alpha$ and He I 5875 we find in all cases evidence for blended emission or absorption profiles of complex morphology (Fig. 1). The HeI 5875 profiles are usually broad and shallow, being the AU Mon HeI 5875 profile exceptionally deep among Galactic DPVs.

LP Ara (HD 328568, 2MASS J16400178-4639348, B = 10.48, B-V = 0.28) is classified as an eclipsing binary of $\beta$ Lyr type in SIMBAD (simbad.u-strasbg.fr/simbad/). Spectral types B8+[A8] and mass ratio $q=0.090$ were given by Svechnikov \& Kuznetsova (1990). From modeling of photometric observations made with the INTEGRAL/OMC camera, Zasche (2010) found a semidetached system with orbital period $P_{o}=8.53282038 \mathrm{~d}, i=$ $77.1^{\circ}, q=0.2$, ratio between stellar temperatures and radii $T_{1} / T_{2}=1.143$ and $R_{1} / R_{2}=$ 1.135 and no third light. The above authors did not correct their observations for the additional long photometric cycle $P_{l}=273$ days reported by Michalska et al. (2009).

We compared the spectrum taken near the long cycle maximum at $\Phi_{o}=0.96$ with a grid of synthetic model spectra in a region deployed of $\mathrm{HI}$ and He I lines. We find the best fit for the secondary star with the model $T_{\text {eff }}=9500 \mathrm{~K}, \log \mathrm{g}=3.0$ and $v_{2}$ sini $=$ $65 \mathrm{~km} / \mathrm{s}$. We modeled the ASAS-3 light curve and radial velocity of LP Ara with a Wilson-Devinney code obtaining $P_{o}=8.53295 \mathrm{~d}, T_{1}=16400 \mathrm{~K}, q=0.30, i=83.9^{\circ}$, orbital separation $a=41.1 R_{\odot}$, mass funtion $f(m)=5.70 \pm 0.36, M_{1}=9.84 M_{\odot}, M_{2}=$ $2.98 M_{\odot}, R_{1}=5.3 R_{\odot}, R_{2}=11.6 R_{\odot}, \log g_{1}=4.0, \log g_{2}=2.8$ and $V$-band luminosity ratio $k=L_{1} / L_{2}=1.50$. Typical errors of derived physical parameters are $\approx 10-20 \%$. LP Ara is a double lined spectroscopic binary, however according to the present state of our analysis, only lines from the secondary component strictly follow the orbital motion. The use of ASAS-3 photometry corrected for long period changes yields a model free from systematic effects within $5 \%$ accuracy. If third light or additional structures do exist they contribute below $5 \%$ to the total orbital light. 


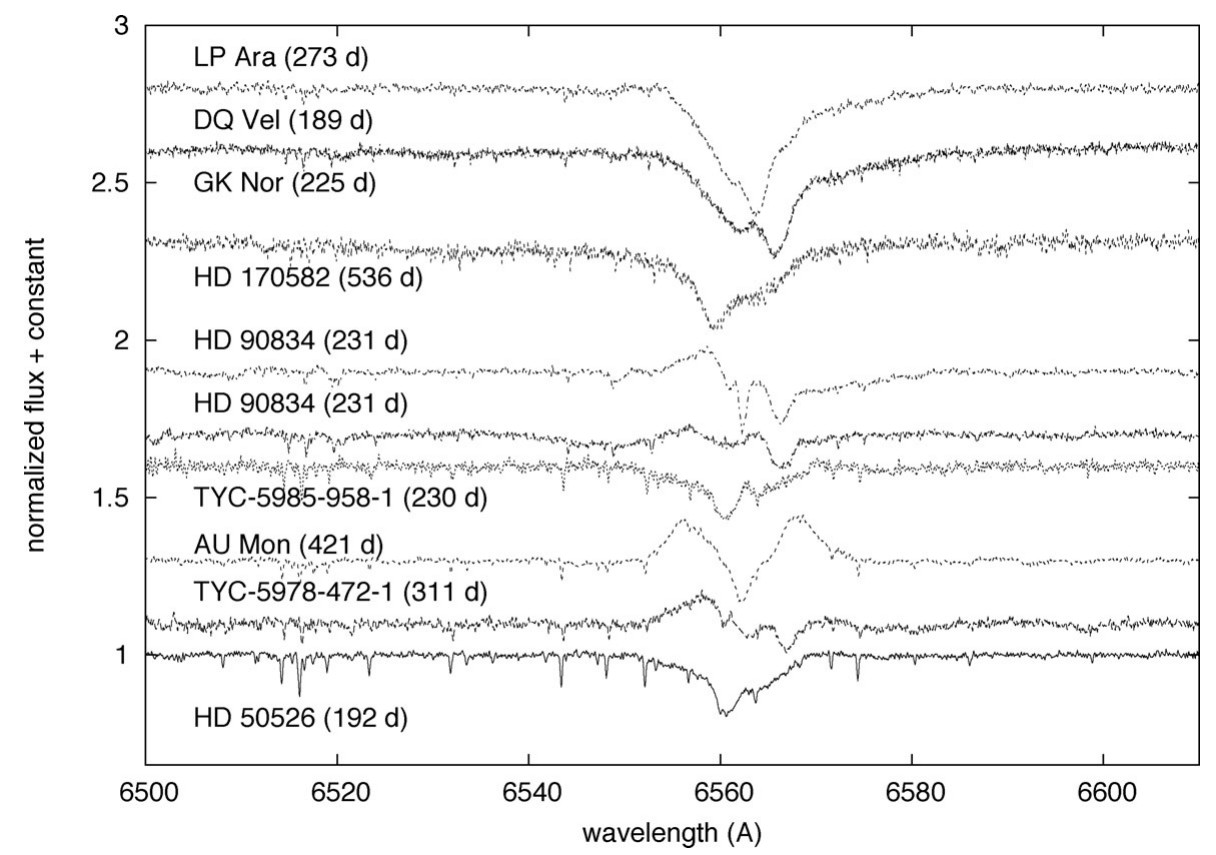

Figure 1. Spectra of Galactic DPVs around $\mathrm{H} \alpha$ at randomly selected orbital phases. The long period, found by us from a study of ASAS-3 ligth curves, is given in parenthesis. Two spectra of HD 90834 illustrate line profile variability. Sharp absorption features are telluric lines.

\section{Conclusions}

DPV H $\alpha$ profiles are complex and usually show asymmetric absorption/emission features varying with the orbital period as well as with the long cycle. This fact suggests that often the line emission region is not disc-like, but more as an irregular structure, a fact already noted for V 393 Sco (Mennickent et al. 2010). The photometric regularity of DPVs (Michalska et al. 2009) place them apart from active Algols (W Serpentids). The rotational velocities of emitting material in some DPVs are much larger than expected for Keplerian orbits around B-type primaries. The system parameters for LP Ara fit the global scheme of low mass ratios found in other DPVs, e.g. OGLE LMC-SC8-125836 and V 393 Scorpii (Mennickent et al. 2008, 2010) and AU Mon (Desmet et al. 2010).

\section{References}

Desmet, M., Frémat, Y., Baudin, F., \& Harmanec, P. et al. 2010, MNRAS 401, 418

Mennickent, R. E., Cidale, L., Díaz, M., \& Pietrzyński, G. et al. 2005, MNRAS 357, 1219

Mennickent, R. E. \& Kołaczkowski, Z. 2009, Binaries: Key to Comprehension of the Universe, ASP-CS, in press, arXiv0908:3900

Mennickent, R. E., Kołaczkowski, Z., Graczyk, D., \& Ojeda, J. 2010, MNRAS 405, 1947

Mennickent, R. E., Kołaczkowski, Z., Michalska, G., \& Pietrzyński, G. et al. 2008, MNRAS 389, 1605

Mennickent, R. E., Pietrzyński, G., Diaz, M., \& Gieren, W. 2003, A\&̛A 399, L47

Michalska, G., Mennickent, R. E., Kołaczkowski, Z. \& Djurašević, G. 2009, ASP-CS, arXiv:0910.4359

Svechnikov, M. A. \& Kuznetsova, E. F. 1990, Katalog priblizhennykh fotometricheskikh $i$ absoliutnykh elementov zatmennykh peremennykh zvezd (Sverdlovsk: Izd-vo Ural'skogo universiteta)

Zasche, P. 2010, New Astron. 15, 150 